\title{
On the condition number of Vandermonde matrices with pairs of nearly-colliding nodes
}

\author{
Stefan Kunis ${ }^{1} \cdot$ Dominik Nagel $^{1}$
}

Received: 28 October 2019 / Accepted: 24 June 2020 / Published online: 21 July 2020

(C) The Author(s) 2020, corrected publication September 2020

\begin{abstract}
We prove upper and lower bounds for the spectral condition number of rectangular Vandermonde matrices with nodes on the complex unit circle. The nodes are "off the grid," pairs of nodes nearly collide, and the studied condition number grows linearly with the inverse separation distance. Such growth rates are known in greater generality if all nodes collide or for groups of colliding nodes. For pairs of nodes, we provide reasonable sharp constants that are independent of the number of nodes as long as non-colliding nodes are well-separated.
\end{abstract}

Keywords Vandermonde matrix · Colliding nodes · Condition number . Frequency analysis · Super resolution

Mathematics Subject Classification (2010) $15 \mathrm{~A} 18 \cdot 65 \mathrm{~T} 40 \cdot 42 \mathrm{~A} 15$

\section{Introduction}

Vandermonde matrices with complex nodes appear in polynomial interpolation problems and many other fields of mathematics (see, e.g., the introduction of [2] and its references). In this paper, we are interested in rectangular Vandermonde matrices with nodes on the complex unit circle and with a large polynomial degree. These matrices generalize the classical discrete Fourier matrices to non-equispaced nodes

The original article was revised: Figure 2 image is correctly presented in this paper.

Stefan Kunis

skunis@uos.de

Dominik Nagel

dnagel@uos.de

1 Institute of Mathematics and Research Center of Cellular Nanoanalytics, Osnabrück University, Osnabrueck, Germany 
and the involved polynomial degree is also called bandwidth. The condition number of those matrices has recently become important in the context of stability analysis of super-resolution algorithms like Prony's method $[6,15]$, the matrix pencil method $[12,18]$, the ESPRIT algorithm [20, 21], and the MUSIC algorithm [17, 22]. If the nodes of such a Vandermonde matrix are all well-separated, with minimal separation distance greater than the inverse bandwidth, bounds on the condition number are established for example in [2, 5, 14, 18].

If nodes are nearly colliding, i.e., their distance is smaller than the inverse bandwidth, the behavior of the condition number is not yet fully understood. The seminal paper [9] coined the term (inverse) super-resolution factor for the product of the bandwidth and the separation distance of the nodes. For $M$ nodes on a grid, the results in $[7,9]$ imply that the condition number grows like the super-resolution factor raised to the power of $M-1$ if all nodes nearly collide. More recently, the practically relevant situation of groups of nearly colliding nodes was studied in $[1,4,16,19]$. In different setups and oversimplifying a bit, all of these refinements are able to replace the exponent $M-1$ by the smaller number $m-1$, where $m$ denotes the number of nodes that are in the largest group of nearly colliding nodes. The authors of [1, 19] focus on quite specific quantities in an optimization approach and in the so-called Prony mapping, respectively. In contrast, the condition number or the relevant smallest singular value of Vandermonde matrices with "off the grid" nodes on the unit circle is studied in [4, 16]. While [4] provided the exponent $m-1$ for the first time, the proof technique leads to quite pessimistic constants and more restrictively asks all nodes (including the well-separated ones) to be within a tiny arc of the unit circle. More recently, the second version of [16] provided a quite general framework and reasonable sharp constants, but involves a technical condition which prevents the separation distance from going to zero for a fixed number of nodes and a fixed bandwidth.

Here, we present upper and lower bounds for the condition number of Vandermonde matrices with pairs of nearly colliding nodes, i.e., the special case $m=2$. We achieve the expected linear order and all constants are reasonably sharp and absolute. In contrast to the more general quoted results $[4,16]$, the nodes can be placed on the full unit circle and the separation distance is allowed to approach zero. Our mild technical conditions, which seem to be artifacts of our proof technique, are:

(i) A logarithmic growth in the separation distance of the well-separated nodes (which can be dropped at a price of a larger constant for the condition number estimate),

(ii) A uniformity condition that colliding nodes behave similarly (they have the same separation distance up to a predefined constant), and

(iii) An a priori upper bound on the separation distance of the colliding nodes.

The outline of this paper is as follows: Section 2 fixes the notation, recalls results for the case of well-separated nodes, and provides lower bounds for the condition number. In Section 3, we establish upper bounds for nodes that are well-separated from each other except for one pair of nodes that is nearly colliding. Section 4 goes one step further and studies the more general case where an arbitrary number of pairs of nodes nearly collide. Theoretical and numerical comparisons with $[3,4,8,16]$ can be found at the end of Section 4 and in Section 5. 


\section{Preliminaries}

Let $\mathbb{T}:=\{z \in \mathbb{C}:|z|=1\}$ be the complex torus and nodes $\left\{z_{1}, \ldots, z_{M}\right\} \subset \mathbb{T}$ be parametrized by $z_{j}=\mathrm{e}^{-2 \pi \mathrm{i} t_{j}}, j=1 \ldots, M$, such that $t_{1}<\cdots<t_{M} \in[0,1)$. We fix a degree $n \in \mathbb{N}$ so that $N:=2 n+1>M$ and set up the rectangular Vandermonde matrix:

$$
A:=\left(z_{j}^{k}\right)_{\substack{j=1, \ldots, M \\
|k| \leq n}}=\left(\begin{array}{cccccccc}
z_{1}^{-n} & \cdots & z_{1}^{-1} & 1 & z_{1}^{1} & \cdots & z_{1}^{n} \\
\vdots & & \vdots & & \vdots & & \\
z_{M}^{-n} & \cdots & z_{M}^{-1} & 1 & z_{M}^{1} & \cdots & z_{M}^{n}
\end{array}\right) \in \mathbb{C}^{M \times N} .
$$

The Dirichlet kernel $D_{n}: \mathbb{R} \rightarrow \mathbb{R}$ is given by:

$$
D_{n}(t):=\sum_{k=-n}^{n} \mathrm{e}^{2 \pi \mathrm{i} k t}= \begin{cases}N, & t \in \mathbb{Z} \\ \frac{\sin (N \pi t)}{\sin (\pi t)}, & \text { otherwise }\end{cases}
$$

so that

$$
K:=A A^{*}=\left(D_{n}\left(t_{i}-t_{j}\right)\right)_{i, j=1}^{M} \in \mathbb{R}^{M \times M} .
$$

The matrix $K$ is symmetric positive definite and the spectral condition number

$$
\operatorname{cond}(A):=\frac{\sigma_{\max }(A)}{\sigma_{\min }(A)}=\sqrt{\|K\|\left\|K^{-1}\right\|}
$$

is finite since all nodes are distinct (here and throughout the paper $\|K\|:=$ $\sup \{\|K x\|:\|x\|=1\}$ with $\|x\|^{2}:=\sum_{k}\left|x_{k}\right|^{2}$ ). On the other hand, if two nodes are equal, then two rows of $A$ are the same and by continuity the condition number diverges if two nodes collide. The (wrap around) distance of two nodes is given by:

$$
\left|t_{j}-t_{\ell}\right|_{\mathbb{T}}:=\min _{r \in \mathbb{Z}}\left|t_{j}-t_{\ell}+r\right| \text {. }
$$

and we introduce the normalized separation distance of the node set as:

$$
\tau:=N \min _{j \neq \ell}\left|t_{j}-t_{\ell}\right|_{\mathbb{T}} .
$$

We call the case $\tau=1$ critical separation, i.e., $\min _{j \neq \ell}\left|t_{j}-t_{\ell}\right|_{\mathbb{T}}=\frac{1}{N}$, and the cases $\tau \leq 1$ and $\tau>1$ nearly colliding and well-separated, respectively. Figure 1 illustrates the situation for 4 nodes on the unit circle. The parameter $\rho_{\text {min }}$ describes a minimum separation distance of involved non-colliding nodes assumed in the theorems.

A reasonable result for well-separated nodes is as follows.

Theorem 2.1 $[2,18]$ Let $A$ be a Vandermonde matrix as in (2.1) with $\tau>1$, then

$$
N\left(1-\frac{1}{\tau}\right) \leq \sigma_{\min }^{2}(A) \leq N \leq \sigma_{\max }^{2}(A) \leq N\left(1+\frac{1}{\tau}\right) .
$$

In particular, we have

$$
\operatorname{cond}(A)^{2} \leq 1+\frac{2}{\tau-1}
$$




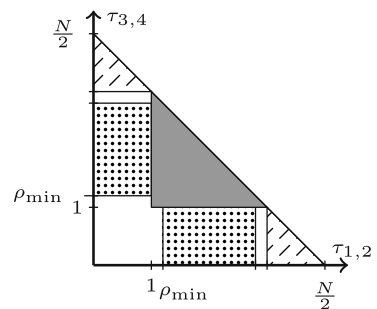

(a) Theorem 3.6

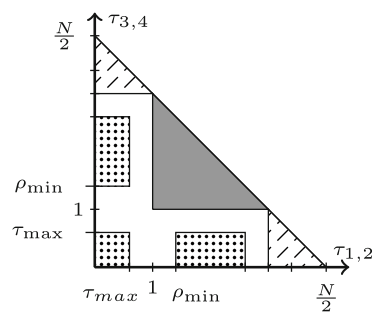

(b) Theorem 4.7

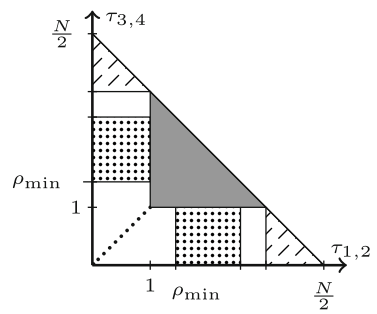

(c) Theorem 4.9

Fig. 1 Sketch of four-node configurations, $t_{1}<t_{2}<t_{3}<t_{4} \in[0,1), t_{1}=0, t_{3}=1 / 2, N$ large enough, $\tau_{1,2}:=N\left|t_{1}-t_{2}\right|_{\mathbb{T}}, \tau_{3,4}:=N\left|t_{3}-t_{4}\right|_{\mathbb{T}}$. dotted, Theorem can be applied; filled, well-separated; lined, 3 nearly colliding nodes; empty areas, at most 2 nearly coll. nodes, but not covered by results

and this implies $\|K\| \leq N+N / \tau$ and $\left\|K^{-1}\right\|=\left\|A^{\dagger}\right\|^{2} \leq(N-N / \tau)^{-1}$, where $A^{\dagger}:=A^{*}\left(A A^{*}\right)^{-1}$ denotes the Moore-Penrose pseudo inverse of $A$.

We note in passing that the above lower bound on the smallest singular value is an improvement of [18] by [2] and that [18] and [8] allow to replace $\frac{1}{\tau}$ in the upper and the lower bounds by $\frac{1}{\tau}-\frac{1}{N}$, respectively. Moreover, we have the following lower bound on the condition number. This already shows that the upper bound for well-separated nodes is quite sharp and provides the benchmark for nearly colliding nodes.

Theorem 2.2 (Lower bound) Let $A$ be a Vandermonde matrix as in (2.1), then:

$$
\sigma_{\min }^{2}(A) \leq N-\left|D_{n}(\tau / N)\right| \leq N \leq N+\left|D_{n}(\tau / N)\right| \leq \sigma_{\max }^{2}(A) .
$$

In particular, we have:

$$
\operatorname{cond}(A)^{2} \geq 1+\frac{2}{\pi \tau-1}
$$

for $\tau \in \mathbb{N}+\frac{1}{2}$, uniformly in $N$ and almost matching the above upper bound.

For nearly colliding nodes, we have:

$$
\operatorname{cond}(A)^{2} \geq \frac{12}{\pi^{2} \tau^{2}}-1 \geq \frac{1}{\tau^{2}}
$$

for $\tau \leq \sqrt{12 / \pi^{2}-1} \approx 0.46$ and $\operatorname{cond}(A) \geq \sqrt{6} / \pi \tau \approx 0.77 / \tau$ for all $\tau \leq 1$.

Proof Without loss of generality, let $t_{2}-t_{1}=\tau / N$ and consider the upper left $2 \times 2$ block in:

$$
K=\left(\begin{array}{cc}
C & * \\
* & *
\end{array}\right), \quad C:=\left(\begin{array}{cc}
D_{n}(0) & D_{n}(\tau / N) \\
D_{n}(\tau / N) & D_{n}(0)
\end{array}\right) .
$$

We apply Lemma A.5, and get:

$\operatorname{cond}(A)^{2}=\frac{\lambda_{\max }(K)}{\lambda_{\min }(K)} \geq \frac{\lambda_{\max }(C)}{\lambda_{\min }(C)}=\frac{D_{n}(0)+\left|D_{n}(\tau / N)\right|}{D_{n}(0)-\left|D_{n}(\tau / N)\right|}=1+\frac{2\left|D_{n}(\tau / N)\right|}{N-\left|D_{n}(\tau / N)\right|}$, and Lemma A.1 yields the assertion. 


\section{Nodes with one nearly colliding pair}

Definition 3.1 Let $M \geq 2$ and $0=t_{1}<\cdots<t_{M} \in[0,1)$ such that:

$$
\begin{array}{ll}
\left|t_{1}-t_{2}\right|_{\mathbb{T}}=\frac{\tau}{N}, & 0<\tau \leq 1, \\
\left|t_{j}-t_{\ell}\right|_{\mathbb{T}} \geq \frac{\rho}{N}, j \neq \ell, \ell \geq 3, & 1<\rho<\infty,
\end{array}
$$

then $\left\{t_{1}, \ldots, t_{M}\right\}$ is called a set of nodes with one nearly colliding pair; see Fig. 2 for an illustration. Due to periodicity, the choice $t_{1}=0$ and $\left|t_{1}-t_{2}\right|_{\mathbb{T}}=\frac{\tau}{N}$ is without loss of generality.

Now, we estimate an upper bound on the condition number of the Hermitian matrix $K$ by bounding $\|K\|$ directly and applying Lemma A.4 to $K^{-1}$ before bounding $\left\|K^{-1}\right\|$. For that, we introduce some notation for abbreviation.

Definition 3.2 We define $a_{1}:=\left(z_{1}^{k}\right)_{|k| \leq n} \in \mathbb{C}^{1 \times N}$ and $A_{2}:=\left(z_{j}^{k}\right)_{\substack{j=2, \ldots, M \\|k| \leq n}} \in$ $\mathbb{C}^{(M-1) \times N}$ so that with:

$$
a_{1} a_{1}^{*}=N, \quad K_{2}:=A_{2} A_{2}^{*} \quad \text { and } \quad b:=A_{2} a_{1}^{*}=\left(\begin{array}{c}
D_{n}(\tau / N) \\
D_{n}\left(t_{3}\right) \\
\vdots \\
D_{n}\left(t_{M}\right)
\end{array}\right) \text {, }
$$

we have the partitioning:

$$
A=\left(\begin{array}{l}
a_{1} \\
A_{2}
\end{array}\right) \quad \text { and } \quad K=\left(\begin{array}{cc}
N & b^{*} \\
b & K_{2}
\end{array}\right),
$$

where $A_{2}$ is a Vandermonde matrix with nodes that are at least $\frac{\rho}{N}$ separated.

Lemma 3.3 Under the conditions of Definition 3.1 and for $\rho \geq 6$, we have:

$$
\|K\| \leq 2.3 N
$$

Proof The key idea is to see the set of nodes as a union of two well-separated subsets and use the existing bounds for these. In contrast to the next chapter, here, one of the sets only consists of a single node. We start by noting that Theorem 2.1 and (3.1)

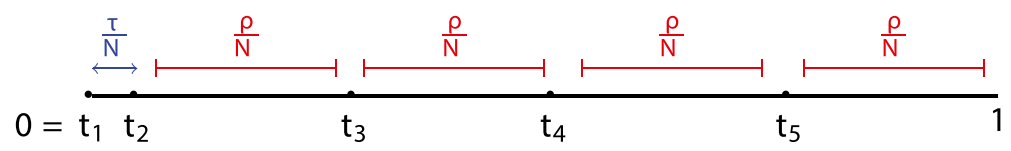

Fig. 2 Example of a node set with $M=5$ satisfying Def. 3.1 
yield $\|b\|^{2} \leq\left\|a_{1}\right\|^{2}\left\|A_{2}\right\|^{2}=N\left\|K_{2}\right\|$. Together with the decomposition (3.2), the triangle inequality, Lemma A.6, and Theorem 2.1, we obtain:

$$
\|K\| \leq\left\|\left(\begin{array}{cc}
N & 0 \\
0 & K_{2}
\end{array}\right)\right\|+\left\|\left(\begin{array}{cc}
0 & b^{*} \\
b & 0
\end{array}\right)\right\| \leq\left\|K_{2}\right\|+\|b\| \leq N\left(\frac{\rho+1}{\rho}+\sqrt{\frac{\rho+1}{\rho}}\right) .
$$

Lemma 3.4 Under the conditions of Definition 3.1 and with $b$ as in (3.1), we have:

$$
b=K_{2} e_{1}+r
$$

where $e_{1} \in \mathbb{R}^{(M-1)}$ denotes the first unit vector and:

$$
\|r\|^{2} \leq\left(N-D_{n}(\tau / N)\right)^{2}+N^{2} \tau^{2}\left(\frac{\pi^{4}}{12 \rho^{2}}+\frac{1.21 \pi}{\rho^{3}}+\frac{\pi^{4}}{180 \rho^{4}}\right) .
$$

Proof The vector $b$ can be approximated by the first column of $K_{2}$ in the sense that:

$$
b=\left(\begin{array}{c}
D_{n}(\tau / N) \\
D_{n}\left(t_{3}\right) \\
\vdots \\
D_{n}\left(t_{M}\right)
\end{array}\right)=\left(\begin{array}{c}
D_{n}(0) \\
D_{n}\left(t_{3}-\tau / N\right) \\
\vdots \\
D_{n}\left(t_{M}-\tau / N\right)
\end{array}\right)+\left(\begin{array}{c}
r_{1} \\
\vdots \\
r_{M-1}
\end{array}\right)
$$

We have $\left|r_{1}\right|=N-D_{n}(\tau / N)$ and for $j=2, \ldots, M-1$ the mean value theorem yields:

$$
\left|r_{j}\right|=\left|D_{n}\left(t_{j+1}\right)-D_{n}\left(t_{j+1}-\tau / N\right)\right|=\left|D_{n}^{\prime}\left(\xi_{j}\right)\right| \frac{\tau}{N}, \quad \xi_{j} \in\left(\left|t_{j+1}-\frac{\tau}{N}\right|_{\mathbb{T}},\left|t_{j+1}\right|_{\mathbb{T}}\right) .
$$

Note that, in the worst case, half of the nodes can be as close as possible (under the assumed separation condition) to $t_{2}$ not only on its right but also on its left. Hence, for $j=2, \ldots,\left\lceil\frac{M}{2}\right\rceil, \xi_{j} \geq \frac{(j-1) \rho}{N}$ and Lemma A.1 lead to:

$$
\left|r_{j}\right| \leq N\left(\frac{\pi}{2 N\left|\xi_{j}\right|}+\frac{1}{2 N^{2}\left|\xi_{j}\right|^{2}}\right) \tau \leq N\left(\frac{\pi}{2(j-1) \rho}+\frac{1}{2(j-1)^{2} \rho^{2}}\right) \tau .
$$

Thus, for all nodes, we get:

$$
\sum_{j=2}^{M-1}\left|r_{j}\right|^{2} \leq 2 \sum_{j=2}^{\lceil M / 2\rceil}\left|r_{j}\right|^{2} \leq N^{2} \tau^{2}(\frac{\pi^{2}}{2 \rho^{2}} \underbrace{\sum_{j=1}^{\infty} \frac{1}{j^{2}}}_{=\frac{\pi^{2}}{6}}+\frac{\pi}{\rho^{3}} \underbrace{\sum_{j=1}^{\infty} \frac{1}{j^{3}}}_{\leq 1.21}+\frac{1}{2 \rho^{4}} \underbrace{\sum_{j=1}^{\infty} \frac{1}{j^{4}}}_{=\frac{\pi^{4}}{90}}) .
$$

Lemma 3.5 Under the conditions of Definition 3.1 and for $\rho \geq 5$, we have:

$$
\left\|K^{-1}\right\| \leq \frac{C(\rho)}{N \tau^{2}}
$$


where

$$
C(\rho)=\left(\frac{2 \rho-1}{\rho-1}+\sqrt{\frac{\rho}{\rho-1}}\right)\left[2-\frac{\rho}{\rho-1}\left(1+\frac{\pi^{4}}{12 \rho^{2}}+\frac{1.21 \pi}{\rho^{3}}+\frac{\pi^{4}}{180 \rho^{4}}\right)\right]^{-1} .
$$

Proof We consider $K$ decomposed as in (3.2) and apply Lemma A.4 with respect to $K_{2}$ to obtain:

$$
K^{-1}=\left(\begin{array}{cc}
I & 0 \\
-K_{2}^{-1} b & I
\end{array}\right)\left(\begin{array}{cc}
\left(N-b^{*} K_{2}^{-1} b\right)^{-1} & 0 \\
0 & K_{2}^{-1}
\end{array}\right)\left(\begin{array}{cc}
I & -b^{*} K_{2}^{-1} \\
0 & I
\end{array}\right)
$$

and thus,

$$
\left\|K^{-1}\right\| \leq\left\|\left(\begin{array}{cc}
I & 0 \\
-K_{2}^{-1} b & I
\end{array}\right)\right\|^{2} \max \left\{\left\|K_{2}^{-1}\right\|,\left\|\left(N-b^{*} K_{2}^{-1} b\right)^{-1}\right\|\right\} .
$$

First of all, we establish an upper bound for the norm of the triangular matrix. Equation (3.1) and Theorem 2.1 imply:

$$
\left\|K_{2}^{-1} b\right\|=\left\|\left(A_{2} A_{2}^{*}\right)^{-1} A_{2} a_{1}^{*}\right\| \leq\left\|A_{2}^{\dagger}\right\|\left\|a_{1}\right\| \leq \sqrt{\frac{\rho}{\rho-1}} .
$$

Together with Lemma A.6, we obtain:

$$
\left\|\left(\begin{array}{cc}
I & 0 \\
-K_{2}^{-1} b & I
\end{array}\right)\right\|^{2} \leq 1+\left\|K_{2}^{-1} b\right\|+\left\|K_{2}^{-1} b\right\|^{2} \leq \frac{2 \rho-1}{\rho-1}+\sqrt{\frac{\rho}{\rho-1}} .
$$

The next step is to bound $\left(N-b^{*} K_{2}^{-1} b\right)^{-1}$. Lemma 3.4 yields:

$$
b^{*} K_{2}^{-1} b=\left(K_{2} e_{1}+r\right)^{*} K_{2}^{-1}\left(K_{2} e_{1}+r\right)=2 D_{n}(\tau / N)-D_{n}(0)+r^{*} K_{2}^{-1} r .
$$

Applying the second part of Lemma 3.4, Lemma A.1, and Theorem 2.1 yields:

$$
\begin{aligned}
N-b^{*} K_{2}^{-1} b & \geq 2\left(N-D_{n}(\tau / N)\right)-\|r\|^{2}\left\|K_{2}^{-1}\right\| \\
& \geq\left(N-D_{n}(\tau / N)\right)\left(2-\left(N-D_{n}(\tau / N)\right)\left\|K_{2}^{-1}\right\|\right)-\left\|K_{2}^{-1}\right\| \sum_{j=2}^{M-1}\left|r_{j}\right|^{2} \\
& \geq N \tau^{2}\left(2-N\left\|K_{2}^{-1}\right\|\right)-\left\|K_{2}^{-1}\right\| N^{2} \tau^{2}\left(\frac{\pi^{4}}{12 \rho^{2}}+\frac{1.21 \pi}{\rho^{3}}+\frac{\pi^{4}}{180 \rho^{4}}\right) \\
& \geq N \tau^{2}\left[2-\frac{\rho}{\rho-1}\left(1+\frac{\pi^{4}}{12 \rho^{2}}+\frac{1.21 \pi}{\rho^{3}}+\frac{\pi^{4}}{180 \rho^{4}}\right)\right] .
\end{aligned}
$$

For $\rho \geq 5$, the most inner bracketed term takes values in $(1,1.4)$ such that the square bracketed term is positive. Forming the reciprocal gives the result, since Theorem 2.1 also implies:

$$
N\left\|K_{2}^{-1}\right\| \leq \frac{\rho}{\rho-1} \leq \frac{\rho-1}{\rho-2} \leq\left[2-\frac{\rho}{\rho-1}(1+\ldots)\right]^{-1} .
$$


Theorem 3.6 (Upper bound) Under the conditions of Definition 3.1 with $\rho \geq \rho_{\min }=$ 6, we have:

$$
\operatorname{cond}(A) \leq \frac{4}{\tau}
$$

Proof The bound follows from Lemmata 3.3 and 3.5 with $C(\rho) \leq C(6) \leq 6.5$.

Lower and upper bounds in Theorems 2.2 and 3.6 yield:

$$
\frac{1}{\tau} \leq \operatorname{cond}(A) \leq \frac{4}{\tau}
$$

for $\tau \leq 0.46$ and $6 \leq \rho$. The condition on $\rho$ implies that for specific configurations of $M$ nodes, our result becomes effective as early as $N \approx 6 M$-this is in contrast to the results $[4,16]$, where $N$ has to be much larger.

Remark 3.7 (Constants) Some comments regarding what is lost during our proof:

(i) The constant in Lemma 3.3 is a numerical value for all $\rho \geq 6$, indeed the proof is valid for all values $\rho>1$. The case $M=2$ shows that Lemmata 3.3 and 3.4 are reasonably sharp since in this case $\|K\|=N+D_{n}(\tau / N) \geq N\left(2-\pi^{2} \tau^{2} / 6\right)$ and $\|r\|=N-D_{n}(\tau / N) \geq N\left(2-\tau^{2}\right)$; see Lemma A.1 for the two inequalities.

(ii) In Lemma 3.5, the constant $C(\rho)$ is monotone decreasing in $\rho$; see also Fig. 3. It is bounded below by 3 which is due to the relatively crude norm estimate on the block triangular factors in the Schur complement decomposition. Note that the left-hand side in (3.3) is bounded from below by $1+\left\|K_{2}^{-1} b\right\|^{2}$. An additional minor improvement on $C(\rho)$ and on the range of admissible values for $\rho$ can be achieved when applying Lemma A.1 to two factors simultaneously.

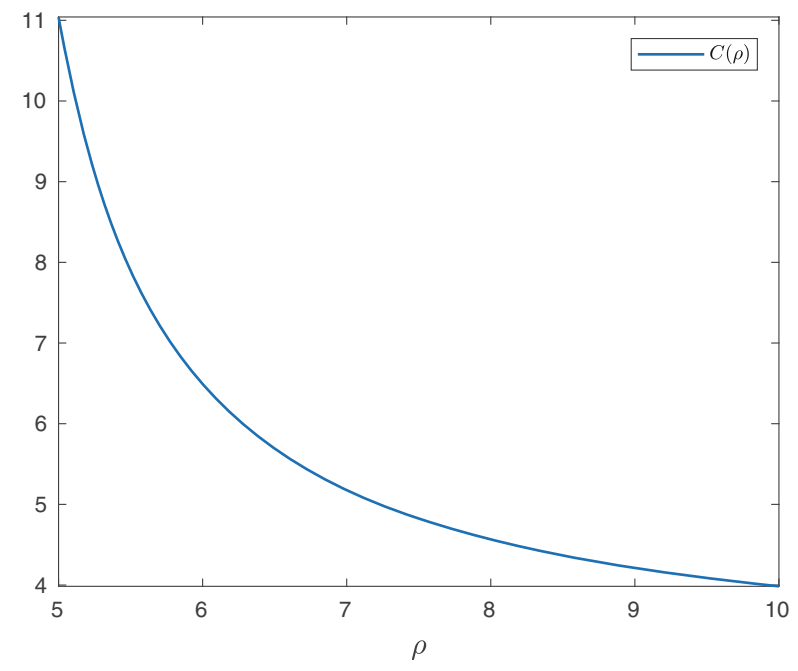

Fig. $3 C(\rho)$ in Lemma 3.5 
Remark 3.8 (Generalizations and limitations) In principle, the suggested Schur complement technique can be generalized to more than two nodes colliding and also to the multivariate case:

(i) Let $M \geq 3$ and $0=t_{1}<\cdots<t_{M} \in[0,1)$ be such that $\left\{t_{1}, t_{2}, t_{3}\right\}$ nearly collide and decompose:

$$
\begin{aligned}
K & =\left(\begin{array}{cc}
K_{1} & B^{*} \\
B & K_{2}
\end{array}\right), \quad K_{1}=\left(\begin{array}{cc}
N & D_{n}\left(t_{1}-t_{2}\right) \\
D_{n}\left(t_{1}-t_{2}\right) & N
\end{array}\right), \\
K_{2} & =\left(D_{n}\left(t_{i}-t_{j}\right)\right)_{i, j=3}^{M} .
\end{aligned}
$$

While it is clear that the Schur complement $K_{1}-B^{*} K_{2}^{-1} B$ is strictly positive definite, establishing a lower bound on its smallest singular value similar to the proofs of Lemmata 3.4 and 3.5 seems considerably harder. Already, the linear approximation in Lemma 3.4 then needs to be replaced by a higher order approximation for the matrix $B$.

(ii) Consider the bivariate case and the Vandermonde matrix:

$$
A=\left(z_{j}^{\gamma}\right)_{\substack{j=1, \ldots, M \\\|\gamma\|_{\infty} \leq n}} \in \mathbb{C}^{M \times N^{2}}
$$

where $z_{j}=\left(x_{j}, y_{j}\right)=\left(\mathrm{e}^{-2 \pi \mathrm{i} u_{j}}, \mathrm{e}^{-2 \pi \mathrm{i} v_{j}}\right) \in \mathbb{T}^{2}, \gamma=(\alpha, \beta) \in \mathbb{Z}^{2}$ is a multiindex, and $z_{j}^{\gamma}:=x_{j}^{\alpha} \cdot y_{j}^{\beta}$. The distance of the nodes $t_{j}=\left(u_{j}, v_{j}\right) \in[0,1)^{2}$ is measured by $\left|t_{j}-t_{\ell}\right|_{\mathbb{T}}:=\min _{r \in \mathbb{Z}^{2}}\left\|t_{j}-t_{\ell}+r\right\|_{\infty}$ and we consider the situation as in Definitions 3.1 and 3.2 with $K=A A^{*}$. Lemma 3.4 can be proven using the bivariate mean value theorem to get $\left|r_{j}\right| \leq N \tau \pi /\left|\xi_{j}\right|_{\mathbb{T}}$, $j=2,3, \ldots, M$, and the packing argument [14, Lem. 4.5] to get:

$$
\|r\|^{2} \leq\left(N^{2}-D_{n}\left(u_{2}\right) D_{n}\left(v_{2}\right)\right)^{2}+\frac{12 \pi^{2} N^{4} \tau^{2}}{(\rho-1)^{2}}(1+\log \lceil\sqrt{M / 6}\rceil) .
$$

We need additional assumptions for Lemma 3.5 to work since results for general well-separated nodes, cf. [15], seem to be too weak. If the nodes $t_{2}, \ldots, t_{M}$ are a subset of equispaced nodes in $\mathbb{T}^{2}$, then [14, Cor. 4.11] yields $\left\|K_{2}^{-1}\right\| \leq$ $(N-N / \rho)^{-2}$. Together with $M \geq 4$ and $\rho \geq 4+2 \log M$, this yields $\left\|K^{-1}\right\| \leq$ $20 / N^{2} \tau^{2}$

\section{Pairs of nearly colliding nodes}

We now study the situation in which the Vandermonde matrix comes from pairs of nearly colliding nodes. 
Definition 4.1 Let $n \in \mathbb{N}, N=2 n+1, c \geq 1$ and let $t_{1}<\cdots<t_{\frac{M}{2}} \in[0,1)$ and $t_{\frac{M}{2}+1}<\cdots<t_{M} \in[0,1)$ for $M \geq 4$ even such that:

$$
\begin{aligned}
& \frac{\tau}{N} \leq\left|t_{j}-t_{j+\frac{M}{2}}\right|_{\mathbb{T}} \leq \frac{c \tau}{N}, \quad j=1, \ldots, \frac{M}{2}, \quad 0<c \tau \leq 1, \\
& \frac{\rho}{N} \leq\left|t_{j}-t_{\ell}\right|_{\mathbb{T}}, \quad j<\ell, \ell \neq j+\frac{M}{2}, \quad 1<\rho<\infty,
\end{aligned}
$$

then $\left\{t_{1}, \ldots, t_{M}\right\}$ is called a set of nodes with pairs of nearly colliding nodes (see Fig. 4 for an illustration). The constant $c$ measures the uniformity of the colliding nodes. For subsequent use, we additionally introduce the following wrap around distance of indices $|j-\ell|^{\prime}:=\min _{r \in \mathbb{Z}}\left|j-\ell+r \frac{M}{2}\right|$ with respect to $\frac{M}{2}$.

Definition 4.2 We define:

$$
A_{1}:=\left(z_{j}^{k}\right)_{\substack{j=1, \ldots, M / 2 \\|k| \leq n}} \in \mathbb{C}^{(M / 2) \times N} \text { and } A_{2}:=\left(z_{j}^{k}\right)_{j=M / 2+1, \ldots, M} \in \mathbb{C}^{(M \mid \leq n) \times N}
$$

so that with $K_{1}:=A_{1} A_{1}^{*}, K_{2}:=A_{2} A_{2}^{*}$, and $B:=A_{2} A_{1}^{*}$ we have the partitioning:

$$
A=\left(\begin{array}{l}
A_{1} \\
A_{2}
\end{array}\right), \quad K=\left(\begin{array}{cc}
K_{1} & B^{*} \\
B & K_{2}
\end{array}\right)
$$

Note that under the assumptions in Definition 4.1 the Vandermonde matrices $A_{1}$ and $A_{2}$ are each corresponding to nodes that are at least $\rho / N$-separated.

The proof technique we use is analogous to the one we used in the case of two nearly colliding nodes. The difference is that we have a matrix $K_{1}$ instead of a scalar and the block $B$ is a matrix instead of a vector. Subsequently, Lemma 4.3 establishes an upper bound on $\|K\|$ and Lemmata 4.4, 4.5, and 4.6 establish an upper bound on $\left\|K^{-1}\right\|$.

Lemma 4.3 Under the conditions of Definition 4.1, we have:

$$
\|K\| \leq 2 N \cdot \frac{\rho+1}{\rho} .
$$

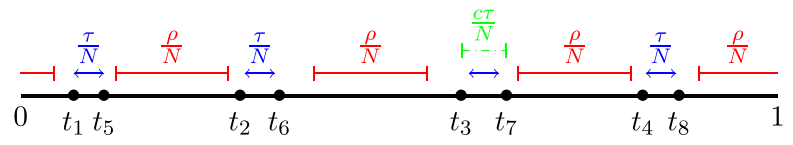

Fig. 4 Example of a node set with $M=8$ satisfying Definition 4.1 
Proof Similar to Lemma 3.3, we start by noting that $\|B\|^{2} \leq\left\|K_{1}\right\|\left\|K_{2}\right\|$. Together with the decomposition (4.1), the triangle inequality, Lemma A.6, and Theorem 2.1, this leads to:

$$
\begin{aligned}
\|K\| & \leq\left\|\left(\begin{array}{cc}
K_{1} & 0 \\
0 & K_{2}
\end{array}\right)\right\|+\left\|\left(\begin{array}{cc}
0 & B^{*} \\
B & 0
\end{array}\right)\right\| \\
& \leq \max \left\{\left\|K_{1}\right\|,\left\|K_{2}\right\|\right\}+\sqrt{\left\|K_{1}\right\|\left\|K_{2}\right\|} \leq 2 N \cdot \frac{\rho+1}{\rho} .
\end{aligned}
$$

Lemma 4.4 Under the conditions of Definition 4.1, $R_{1}:=B-K_{1}$ fulfills:

$$
\left\|R_{1}\right\| \leq N-D_{n}(c \tau / N)+N c \tau\left(\frac{\pi\left(\log \left\lfloor\frac{M}{4}\right\rfloor+1\right)}{\rho}+\frac{\pi^{2}}{6 \rho^{2}}\right) .
$$

Proof The Dirichlet kernel $D_{n}$ is monotone decreasing on $[0,1 / N]$. Hence, for the diagonal entries, we obtain:

$$
\left|\left(R_{1}\right)_{j j}\right|=\left|D_{n}\left(t_{j}-t_{j+\frac{M}{2}}\right)-N\right|=N-D_{n}\left(t_{j}-t_{j+\frac{M}{2}}\right) \leq N-D_{n}(c \tau / N) .
$$

The off-diagonal entries are bounded by the mean value theorem and Lemma A.1 as:

$$
\begin{aligned}
\left|\left(R_{1}\right)_{j \ell}\right| & =\left|D_{n}\left(t_{j}-t_{\ell}\right)-D_{n}\left(t_{j+\frac{M}{2}}-t_{\ell}\right)\right| \\
& \leq\left|D_{n}^{\prime}\left(\xi_{j \ell}\right)\right| \frac{c \tau}{N} \leq N c \tau\left(\frac{\pi}{2 N \xi_{j \ell}}+\frac{1}{2 N^{2} \xi_{j \ell}^{2}}\right),
\end{aligned}
$$

where $\left(\left|t_{j+\frac{M}{2}}-t_{\ell}\right|_{\mathbb{T}},\left|t_{j}-t_{\ell}\right|_{\mathbb{T}}\right) \ni \xi_{j \ell} \geq|j-\ell|^{\prime} \rho / N$ implies:

$$
\left|\left(R_{1}\right)_{j \ell}\right| \leq N c \tau\left(\frac{\pi}{2 \rho|j-\ell|^{\prime}}+\frac{1}{2 \rho^{2}\left(|j-\ell|^{\prime}\right)^{2}}\right)=:\left(\widetilde{R}_{1}\right)_{j \ell}
$$

for $j, \ell=1, \ldots, \frac{M}{2}, j \neq \ell$. Additionally, we set $\left(\widetilde{R}_{1}\right)_{j j}:=N-D_{n}(c \tau / N)$. We bound the spectral norm of $R_{1}$ by the one of the real symmetric matrix $\widetilde{R}_{1}$ using Lemma A. 2 and proceed by:

$$
\left\|R_{1}\right\| \leq\left\|\widetilde{R}_{1}\right\| \leq\left\|\widetilde{R}_{1}\right\|_{\infty} \leq N-D_{n}(c \tau / N)+2 N c \tau \sum_{j=1}^{\left\lfloor\frac{M}{4}\right\rfloor}\left(\frac{\pi}{2 j \rho}+\frac{1}{2 j^{2} \rho^{2}}\right),
$$

from which the assertion follows.

Lemma 4.5 Under the conditions of Definition 4.1, $R_{1}=B-K_{1}$ and $R_{2}:=B-K_{2}$ fulfill:

$$
\left\|2 N I+R_{1}^{*}+R_{2}\right\| \leq 2 D_{n}(\tau / N)+c^{2} \tau^{2} N\left(\frac{\pi^{2}\left(\log \left\lfloor\frac{M}{4}\right\rfloor+1\right)}{\rho}+\frac{\pi^{3}}{3 \rho^{2}}+\frac{2.42}{\rho^{3}}\right) .
$$


Proof First, note that:

$$
\left(R_{1}^{*}+R_{2}\right)_{j \ell}=D_{n}\left(t_{j+\frac{M}{2}}-t_{\ell}\right)+D_{n}\left(t_{j}-t_{\ell+\frac{M}{2}}\right)-D_{n}\left(t_{j+\frac{M}{2}}-t_{\ell+\frac{M}{2}}\right)-D_{n}\left(t_{j}-t_{\ell}\right) .
$$

Monotonicity of the Dirichlet kernel $D_{n}$ on $t \in[0,1 / N]$ gives:

$$
\left|\left(2 N I+R_{1}^{*}+R_{2}\right)_{j j}\right|=2\left|D_{n}\left(t_{j+\frac{M}{2}}-t_{j}\right)\right| \leq 2 D_{n}(\tau / N)
$$

for $j=\ell$. For each fixed off-diagonal entry $j \neq \ell$, the matrix $2 N I$ has no contribution. We write the node $t_{j+M / 2}$ as a perturbation of $t_{j}$ by $h_{j}:=t_{j+M / 2}-t_{j}$ and expand the Dirichlet kernel by its Taylor polynomial of degree 2 in the point $\hat{h}:=t_{j}-t_{\ell}+\frac{h_{j}-h_{\ell}}{2}$. Using:

$$
D_{n}(h)=D_{n}(\hat{h})+D_{n}^{\prime}(\hat{h})(h-\hat{h})+\frac{D_{n}^{\prime \prime}(\xi)}{2}(h-\hat{h})^{2}
$$

for some $\xi \in[\hat{h}, h] \cup[h, \hat{h}]$, the constant term, as well as the linear term, cancels out and we get:

$$
\begin{aligned}
& D_{n}\left(t_{j}+h_{j}-t_{\ell}\right)+D_{n}\left(t_{j}-t_{\ell}-h_{\ell}\right)-D_{n}\left(t_{j}+h_{j}-t_{\ell}-h_{\ell}\right)-D_{n}\left(t_{j}-t_{\ell}\right) \\
= & \frac{1}{8}\left(D_{n}^{\prime \prime}\left(\xi_{1}\right)\left(h_{j}+h_{\ell}\right)^{2}+D_{n}^{\prime \prime}\left(\xi_{2}\right)\left(h_{j}+h_{\ell}\right)^{2}+D_{n}^{\prime \prime}\left(\xi_{3}\right)\left(h_{j}-h_{\ell}\right)^{2}+D_{n}^{\prime \prime}\left(\xi_{4}\right)\left(h_{j}-h_{\ell}\right)^{2}\right) .
\end{aligned}
$$

Lemma A. 1 and $\xi_{1}, \ldots, \xi_{4} \geq|j-\ell|^{\prime} \rho / N$ imply:

$$
\begin{aligned}
\left|\left(R_{1}^{*}+R_{2}\right)_{j \ell}\right| \leq & \frac{N^{3}}{4}\left(\frac{\pi^{2}}{2|j-\ell|^{\prime} \rho}+\frac{\pi}{\left(|j-\ell|^{\prime}\right)^{2} \rho^{2}}+\frac{1}{\left(|j-\ell|^{\prime}\right)^{3} \rho^{3}}\right) \\
& \cdot\left(\left(h_{j}+h_{\ell}\right)^{2}+\left(h_{j}-h_{\ell}\right)^{2}\right)
\end{aligned}
$$

and hence by $h_{j}, h_{\ell} \leq c \tau / N$

$$
\left|\left(2 N I+R_{1}^{*}+R_{2}\right)_{j \ell}\right| \leq N c^{2} \tau^{2}\left(\frac{\pi^{2}}{2|j-\ell|^{\prime} \rho}+\frac{\pi}{\left(|j-\ell|^{\prime}\right)^{2} \rho^{2}}+\frac{1}{\left(|j-\ell|^{\prime}\right)^{3} \rho^{3}}\right) .
$$

The matrix $2 N I+R_{1}^{*}+R_{2}$ is real symmetric so that:

$$
\begin{aligned}
\left\|2 N I+R_{1}^{*}+R_{2}\right\| & \leq\left\|2 N I+R_{1}^{*}+R_{2}\right\|_{\infty} \\
& \leq 2 D_{n}(\tau / N)+2 \sum_{j=1}^{\left\lfloor\frac{M}{4}\right\rfloor} N c^{2} \tau^{2}\left(\frac{\pi^{2}}{2 j \rho}+\frac{\pi}{j^{2} \rho^{2}}+\frac{1}{j^{3} \rho^{3}}\right) \\
& \leq 2 D_{n}(\tau / N)+2 c^{2} \tau^{2} N\left(\frac{\pi^{2}\left(\log \left\lfloor\frac{M}{4}\right\rfloor+1\right)}{2 \rho}+\frac{\pi^{3}}{6 \rho^{2}}+\frac{1.21}{\rho^{3}}\right)
\end{aligned}
$$

and therefore the result holds. 
Lemma 4.6 Under the conditions of Definition 4.1 with $\tau \leq 1 / 2$ and $\rho \geq 2$, such that:

$$
\begin{aligned}
\tilde{C}(\tau, \rho, c, M):= & 2-\frac{c^{2} \pi^{2}\left(\log \left\lfloor\frac{M}{4}\right\rfloor+1\right)}{\rho}-\frac{c^{2} \pi^{3}}{3 \rho^{2}}-\frac{2.42 c^{2}}{\rho^{3}} \\
& -\frac{\rho}{(\rho-1)}\left(\frac{c^{2} \pi^{2}}{6} \tau+\frac{c \pi\left(\log \left\lfloor\frac{M}{4}\right\rfloor+1\right)}{\rho}+\frac{c \pi^{2}}{6 \rho^{2}}\right)^{2}
\end{aligned}
$$

is positive, we have:

$$
\left\|K^{-1}\right\| \leq \frac{C(\tau, \rho, c, M)}{N \tau^{2}}
$$

where

$$
C(\tau, \rho, c, M):=\left(\frac{2 \rho}{\rho-1}+\sqrt{\frac{\rho+1}{\rho-1}}\right) / \tilde{C}(\tau, \rho, c, M) .
$$

Figure 5 visualizes the values of the constant $\tilde{C}(\tau, \rho, c, M)$ with respect to $\rho$ and $\tau$. Please note that $(i)$ increasing the constant $c$ by a factor $\sqrt{2}$ has to be compensated approximately by halving $\tau$ and doubling $\rho$ and (ii) increasing the number of nodes $M$ from 4 to 64 has to be compensated approximately by tripling $\rho$.

Proof We proceed analogously to Lemma 3.5 and apply Lemma A.4 to the matrix $K$ decomposed as in (4.1) and obtain:

$$
\left\|K^{-1}\right\| \leq \max \left\{\left\|K_{1}^{-1}\right\|,\left\|\left(K_{2}-B K_{1}^{-1} B^{*}\right)^{-1}\right\|\right\}\left\|\left(\begin{array}{cc}
I & 0 \\
-B K_{1}^{-1} & I
\end{array}\right)\right\|^{2} .
$$

Definition 4.2 and Theorem 2.1 yield:

$$
\left\|B K_{1}^{-1}\right\| \leq\left\|A_{2}\right\|\left\|A_{1}^{\dagger}\right\| \leq \sqrt{\frac{\rho+1}{\rho-1}},
$$

together with Lemma A.6, we obtain:

$$
\left\|\left(\begin{array}{cc}
I & 0 \\
-B K_{1}^{-1} & I
\end{array}\right)\right\|^{2} \leq 1+\left\|B K_{1}^{-1}\right\|+\left\|B K_{1}^{-1}\right\|^{2} \leq \frac{2 \rho}{\rho-1}+\sqrt{\frac{\rho+1}{\rho-1}} .
$$
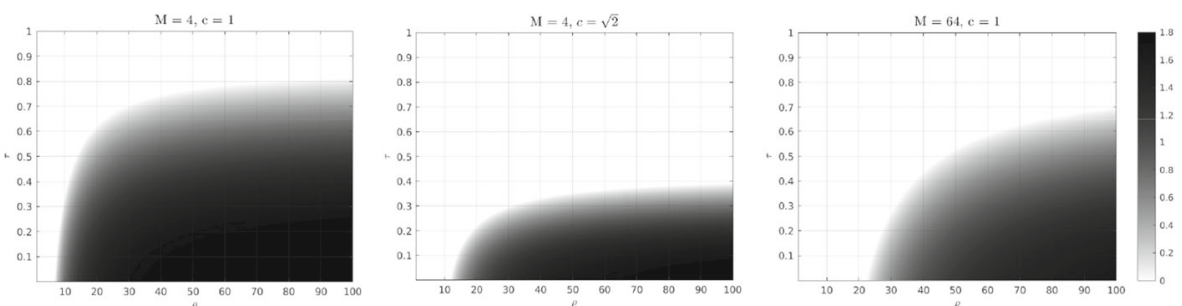

Fig. 5 Values of $\tilde{C}(\tau, \rho, c, M)$ in Lemma 4.6 depending on $\tau$ and $\rho$ for different $M$ and $c$. Negative values are set to zero 
Now, we estimate $\left\|\left(K_{2}-B K_{1}^{-1} B^{*}\right)^{-1}\right\|$, which is done by the following steps:

(i) First, note that $I-A_{1}^{\dagger} A_{1}$ is an orthogonal projector and thus Theorem 2.1 implies:

$$
\left\|K_{2}-B K_{1}^{-1} B^{*}\right\| \leq\left\|A_{2}\right\|\left\|I-A_{1}^{\dagger} A_{1}\right\|\left\|A_{2}^{*}\right\| \leq\left\|A_{2}\right\|^{2}<2 N .
$$

We apply Lemma A.3 with $\eta=2 N$, use the identities $R_{1}=B-K_{1}$ and $R_{2}=B-K_{2}$, apply the triangular inequality, and the sub-multiplicativity of the matrix norm to get:

$$
\begin{aligned}
\left\|\left(K_{2}-B K_{1}^{-1} B^{*}\right)^{-1}\right\| & =\frac{1}{2 N-\left\|2 N I-K_{2}+B K_{1}^{-1} B^{*}\right\|} \\
& \leq \frac{1}{2 N-\left\|2 N I+R_{1}^{*}+R_{2}\right\|-\left\|R_{1}\right\|^{2}\left\|K_{1}^{-1}\right\|}
\end{aligned}
$$

(ii) Lemma 4.5 leads to:

$$
\begin{aligned}
2 N-\left\|2 N I+R_{1}^{*}+R_{2}\right\| \geq & 2\left(N-D_{n}(\tau / N)\right) \\
& -c^{2} \tau^{2} N\left(\frac{\pi^{2}\left(\log \left\lfloor\frac{M}{4}\right\rfloor+1\right)}{\rho}+\frac{\pi^{3}}{3 \rho^{2}}+\frac{2.42}{\rho^{3}}\right) .
\end{aligned}
$$

(iii) We apply Theorem 2.1 and Lemma 4.4 to get:

$$
\left\|R_{1}\right\|^{2}\left\|K_{1}^{-1}\right\| \leq \frac{\rho}{N(\rho-1)}\left[N-D_{n}(c \tau / N)+N c \tau\left(\frac{\pi\left(\log \left\lfloor\frac{M}{4}\right\rfloor+1\right)}{\rho}+\frac{\pi^{2}}{6 \rho^{2}}\right)\right]^{2} .
$$

(iv) We use the estimates for the Dirichlet kernel $N-D_{n}(\tau / N) \geq N \tau^{2}$ in ii) and $N-D_{n}(c \tau / N) \leq N \frac{\pi^{2}}{6} c^{2} \tau^{2}$ in iii) (see Lemma A.1), and insert this in (4.3) to get finally:

$$
\begin{aligned}
\left\|\left(K_{2}-B K_{1}^{-1} B^{*}\right)^{-1}\right\| \leq & \frac{1}{N \tau^{2}}\left[2-\frac{c^{2} \pi^{2}\left(\log \left\lfloor\frac{M}{4}\right\rfloor+1\right)}{\rho}-\frac{c^{2} \pi^{3}}{3 \rho^{2}}-\frac{2.42 c^{2}}{\rho^{3}}\right. \\
& \left.-\frac{\rho}{(\rho-1)}\left(\frac{c^{2} \pi^{2}}{6} \tau+\frac{c \pi\left(\log \left\lfloor\frac{M}{4}\right\rfloor+1\right)}{\rho}+\frac{c \pi^{2}}{6 \rho^{2}}\right)^{2}\right]^{-1} .
\end{aligned}
$$

This upper bound also bounds the maximum in (4.2) since for all $\tau \leq 1 / 2$ and $\rho \geq 2$ together with Theorem 2.1

$$
\left\|K_{1}^{-1}\right\| \leq \frac{2}{N} \leq \frac{1}{2 N \tau^{2}} \leq \frac{1}{N \tau^{2}}[2-\cdots]^{-1} .
$$

Theorem 4.7 (Upper bound) Under the conditions of Definition 4.1 with $M \geq 4$, $\tau \leq \tau_{\max }=\frac{1}{4 c^{2}}$ and $\rho \geq \rho_{\min }=10 c^{2}\left(\log \left\lfloor\frac{M}{4}\right\rfloor+1\right)$, we have:

$$
\operatorname{cond}(A) \leq \frac{5}{\tau}
$$


Proof In Lemma 4.6, the constant $C(\tau, \rho, c, M)$ is monotone increasing in $\tau$ and monotone decreasing in $\rho$. Hence, after plugging in the bounds for $\tau$ and $\rho$ in our assumptions, it is easy to see that the constant $C\left(\frac{1}{4 c^{2}}, 10 c^{2}\left(\log \left\lfloor\frac{M}{4}\right\rfloor+1\right), c, M\right)$ is monotone decreasing in $c$ and $M$, respectively. Therefore, we get $C(\tau, \rho, c, M) \leq$ $C(1 / 4,10,1,4) \leq 11.3$, so that $\left\|K^{-1}\right\| \leq 11.3 N^{-1} \tau^{-2}$. Together with the bound $\|K\| \leq 22 N / 10=2.2 N$ from Lemma 4.3 , we obtain the result.

If each pair of nearly colliding nodes has the same separation distance, i.e., $c=$ 1 , we can improve the upper bound in the sense that restrictions on $\tau$ except for $\tau \leq 1$ can be dropped. In order to obtain the same constant, we have to increase the restrictions on $\rho$ slightly.

Lemma 4.8 Under the conditions of Definition 4.1 with $c=1$, such that:

$$
\begin{aligned}
\tilde{C}(\rho, M):= & 2-\frac{\pi^{2}\left(\log \left\lfloor\frac{M}{4}\right\rfloor+1\right)}{\rho}-\frac{\pi^{3}}{3 \rho^{2}}-\frac{2.42}{\rho^{3}} \\
& -\frac{\rho}{\rho-1}-\frac{2 \pi\left(\log \left\lfloor\frac{M}{4}\right\rfloor+1\right)}{(\rho-1)}-\frac{\pi^{2}}{3 \rho(\rho-1)} \\
& -\frac{\pi^{2}\left(\log \left\lfloor\frac{M}{4}\right\rfloor+1\right)^{2}}{\rho(\rho-1)}-\frac{\pi^{3}\left(\log \left\lfloor\frac{M}{4}\right\rfloor+1\right)}{3 \rho^{2}(\rho-1)}-\frac{\pi^{4}}{36 \rho^{3}(\rho-1)}
\end{aligned}
$$

is positive, we have

$$
\left\|K^{-1}\right\| \leq \frac{C(\rho, M)}{N \tau^{2}}
$$

where $C(\rho, M):=\left(\frac{2 \rho}{\rho-1}+\sqrt{\frac{\rho+1}{\rho-1}}\right) / \tilde{C}(\rho, M)$.

Proof The proof is analogous to that of Lemma 4.6, the only difference is in step (iv). Setting $c=1$ in (ii) and (iii), expanding the squared bracket in (iii) and inserting this into (4.3) leads to:

$$
\begin{aligned}
& \left\|\left(K_{2}-B K_{1}^{-1} B^{*}\right)^{-1}\right\| \leq\left[2\left(N-D_{n}(\tau / N)\right)\right. \\
& -N \tau^{2}\left(\frac{\pi^{2}\left(\log \left\lfloor\frac{M}{4}\right\rfloor+1\right)}{\rho}+\frac{\pi^{3}}{3 \rho^{2}}+\frac{2.42}{\rho^{3}}\right)-\frac{\rho}{N(\rho-1)}\left(N-D_{n}(\tau / N)\right)^{2} \\
& -\frac{\rho}{\rho-1} 2 \tau\left(N-D_{n}(\tau / N)\right)\left(\frac{\pi\left(\log \left\lfloor\frac{M}{4}\right\rfloor+1\right)}{\rho}+\frac{\pi^{2}}{6 \rho^{2}}\right) \\
& \left.-N \tau^{2} \frac{\rho}{\rho-1}\left(\frac{\pi^{2}\left(\log \left\lfloor\frac{M}{4}\right\rfloor+1\right)^{2}}{\rho^{2}}+\frac{\pi^{3}\left(\log \left\lfloor\frac{M}{4}\right\rfloor+1\right)}{3 \rho^{3}}+\frac{\pi^{4}}{36 \rho^{4}}\right)\right]^{-1} .
\end{aligned}
$$

In three summands, we can factor out $N-D_{n}(\tau / N)$ and use the estimate $N-$ $D_{n}(\tau / N) \geq N \tau^{2}$, leading to a larger bound after inverting the expression in the end. Afterwards, in the third summand $N-D_{n}(\tau / N)$ is left, for which we use the rough bound $N-D_{n}(\tau / N) \leq N$. In the fourth summand, we use $\tau \leq 1$ for the single $\tau$. The same argument as in (3.4) shows that this also bounds the maximum in (4.2) and we get the result. 
Theorem 4.9 (Upper bound) Under the conditions of Definition 4.1 with $c=1$, $\rho \geq \rho_{\min }=25\left(\log \left\lfloor\frac{M}{4}\right\rfloor+1\right)$, we have:

$$
\operatorname{cond}(A)<\frac{5}{\tau}
$$

Proof Direct inspection gives monotonicity of $C(\rho, M)$ with respect to $\rho$ and also the estimate $C(25(\log \lfloor M / 4\rfloor+1), M) \leq C(25,4) \leq 12$. Hence, $\left\|K^{-1}\right\| \leq$ $12 N^{-1} \tau^{-2}$ and together with the bound $\|K\| \leq 52 N / 25$ from Lemma 4.3 we obtain the result.

Remark 4.10 Due to Lemma A.5, the upper bound from Theorem 4.7 remains valid if nodes are removed. We note in passing that $\sigma_{\min }$ and $\sigma_{\max }$ are monotone increasing with $N$ and thus, condition number estimates for an even number $N$ follow. Lower and upper bounds in Theorems 2.2 and 4.7 finally yield:

$$
\frac{1}{\tau} \leq \operatorname{cond}(A) \leq \frac{5}{\tau} .
$$

The lower bound is tight and the numerical value 5 in the upper bound follows from our proof technique and can be improved (see Fig. 6). The uniformity condition $\tau \leq$ $1 /\left(4 c^{2}\right)$ is artificial and, except for the special cases in Theorems 3.6 and 4.9 , prevents letting $\tau \rightarrow 1$.

Moreover, the technical condition $\rho \geq \rho_{\min }=25\left(\log \left\lfloor\frac{M}{4}\right\rfloor+1\right)$ in Theorem 4.7 is due to the slow decay of the Dirichlet kernel and can be weakened by a preconditioning technique which however leads to a somewhat larger constant in the final result. ${ }^{1}$ The diagonal matrix $D=\operatorname{diag}(1-|k| /(n+1))_{|k| \leq n} \in \mathbb{C}^{N \times N}$ is positive definite with $\|D\| \leq 1$ and thus the Rayleigh-Riesz characterization of the smallest eigenvalue for Hermitian matrices leads to:

$$
\lambda_{\min }\left(A D A^{*}\right)=\min _{x \in \mathbb{C}^{M},\|x\|=1}\left\|D^{1 / 2} A^{*} x\right\|^{2} \leq \min _{x \in \mathbb{C}^{M},\|x\|=1}\left\|A^{*} x\right\|^{2}=\lambda_{\min }(K) .
$$

The entries of the matrix $A D A^{*}$ consist of Fejér kernel evaluations and analogously to Lemmata $4.4,4.5$, and 4.6 this yields (independently of $M$ ):

$$
\begin{gathered}
\left\|K^{-1}\right\| \leq \frac{C(\tau, \rho, c)}{N \tau^{2}}, \quad C(\tau, \rho, c):=\left(\frac{6 \rho^{2}}{3 \rho^{2}-\pi^{2}}+\sqrt{\frac{3 \rho^{2}+\pi^{2}}{3 \rho^{2}-\pi^{2}}}\right) / \tilde{C}(\tau, \rho, c), \\
\tilde{C}(\tau, \rho, c):=\frac{1}{8}\left(2-\frac{4 c^{2} \pi^{4}}{3 \rho^{2}}-\frac{39 c^{2} \pi}{\rho^{3}}-\frac{16 c^{2} \pi^{4}+2 c^{2} \pi^{6}}{45 \rho^{4}}\right. \\
\left.-\frac{3 \rho^{2}}{3 \rho^{2}-\pi^{2}}\left(\frac{2 c^{2} \pi^{2}}{9} \tau+\frac{2 c \pi^{3}}{3 \rho^{2}}+\frac{9.68 c}{\rho^{3}}\right)^{2}\right) .
\end{gathered}
$$

\footnotetext{
${ }^{1}$ We thank one of the peer reviewers for this clever hint.
} 
Under the conditions of Definition 4.1 with $n \geq 1, \tau \leq \tau_{\max }=\frac{1}{4 c^{2}}$ and $\rho \geq \rho_{\min }=$ $11 c^{2}$, we finally have $\operatorname{cond}(A) \leq 14 / \tau$. Note that this approach also allows to drop the logarithmic factor in Remark 3.8 (ii).

The absolute constant 5 in the upper bound of the condition number (or $\tau \sqrt{N}\left\|A^{\dagger}\right\| \leq \sqrt{11.3} \approx 3.4$ ) follows from our proof technique and we give a numerical comparison to the approaches $[3,4,8,16]$ in Fig. 7. A short theoretical comparison including different assumptions on $N, M, \tau$, and $\rho$ is given below.

Remark 4.11 (Comparison to [4]) This approach is more general and allows more than two nodes in a group nearly colliding. The upper bound on the condition number grows quite strongly like $C M^{M}$ with the total number of nodes $M$, cf. [4, Cor. 3.6]. Moreover, the a priori conditions $N \geq 4 M^{3}$ and $\rho \geq 2 M$ (see [4, Cor. 3.6, left ineq. (3.4)]) are much stronger than ours. The uniformity condition $\tau \leq M /(2 c)$ is slightly weaker than ours but nonetheless artificial.

Remark 4.12 (Comparison to [16]) Again, this approach is more general and allows more than two nodes in a group nearly colliding. The upper bound on the condition number grows like $C \sqrt{M}$ with the total number of nodes $M$, cf. [16, Thm. 1 ineq. (2.3), Thm. 2 ineq. (2.7), and ineq. (2.8)]. With minor simplifications, the a priori conditions $N \geq M^{2}$ and:

$$
\rho \geq \begin{cases}C_{1}\left(\frac{M}{\tau}\right)^{1 / 4}, & \text { with } C_{1} \approx 42, \\ C_{2}\left(\frac{M}{\tau}\right)^{1 / 2}, & \text { with } C_{2} \approx 63,\end{cases}
$$

are imposed; see [16, Thm. 1, ineq. (2.2), Thm 2, ineq. (2.5)]. We note that [16, Thm. 1, Thm. 2] places no upper bound except $\tau \leq 1$ but that condition (4.4) is in fact an a priori lower bound on $\tau$ which prevents $\tau \rightarrow 0$ already for moderate fixed $M \geq 3$. Recently, we refined this approach in [13], dropped the mentioned dependencies on $M$ and could weaken the condition (4.4) considerably.

Remark 4.13 (Comparison to [8]) This approach deals with pairs of nearly colliding nodes but differs completely from ours and the ones in $[3,4,16]$, and rather generalizes the construction of certain extremal functions in [18] to pairs of nearly colliding nodes and subsets of them. The proven constant in the upper bound given in [8, Cor. 4.2] is $\tau \sqrt{N}\left\|A^{\dagger}\right\| \leq 9 \sqrt{6} / \pi \approx 7.0$ and thus is slightly larger than ours $(\sqrt{11.3} \approx 3.4)$. Using the stronger assumption on $\tau$ from our setting in the proof of [8, Thm. 3.6] and improving estimates in [8, Eq. (8)] provides the best result $(\approx 1.7)$ for pairs of nearly colliding nodes. The conditions $\tau \leq 1$ and $3 \leq \rho$ are quasi-optimal. Provided all technical results prove right, this approach is superior.

Remark 4.14 (Comparison to [3]) This approach uses a QR-decomposition technique to establish bounds on all singular values of Vandermonde matrices with nearly colliding nodes. Adapted to the case of pairs of nearly colliding nodes, we obtain the following: Let $M \geq 4$ even and $A$ as in Definition 4.1. With respect to the nearly colliding pairs, partition $A^{*}=\left(A_{1}^{*} A_{2}^{*} \ldots A_{M / 2}^{*}\right)$ with $\mathrm{QR}$ decompositions $A_{j}^{*}=Q_{j} R_{j}$ 
and set $Q:=\left(Q_{1} Q_{2} \ldots Q_{M / 2}\right)$. Tracing back all constants in lemmata and proofs for the case of pairwise nearly colliding nodes, we obtain the uniform off-diagonal estimate:

$$
\left|\left(Q^{*} Q\right)_{j, k}\right| \leq 150 / \rho+1079 \tau, \quad j \neq k,
$$

which yields a constant "multiplicative perturbation" in [3, Lem. 5.1] and thus a condition number estimate like Theorem 4.7 or [8] only if $\tau \leq C_{1} / M$ and $C_{2} M \leq \rho$, for some constants $C_{1}, C_{2}$.

However, note that for two nearly colliding pairs $u_{1}<u_{2} \ll v_{1}<v_{2}$, a direct computation (avoiding a so-called limit basis used in [3]) yields the off-diagonal estimate:

$$
\left\|Q_{1}^{*} Q_{2}\right\|_{\mathrm{F}} \leq \frac{116}{N\left(v_{1}-u_{2}\right)}, \quad Q_{1}=Q_{1}\left(u_{1}, u_{2}\right), Q_{2}=Q_{2}\left(v_{1}, v_{2}\right) .
$$

Together with $\rho \geq \frac{27}{23} \cdot 232\left(\log \left\lfloor\frac{M}{4}\right\rfloor+1\right)$ and Lemma A.6, this gives:

$$
\begin{aligned}
\left|1-\lambda_{r}\left(Q^{*} Q\right)\right| & \leq \max _{j} \sum_{\ell=1}^{M / 2}\left\|Q_{j}^{*} Q_{\ell}\right\|_{\mathrm{F}} \leq 2 \sum_{\ell=1}^{\lfloor M / 4\rfloor} \frac{116}{\ell \rho} \\
& \leq \frac{232\left(\log \left\lfloor\frac{M}{4}\right\rfloor+1\right)}{\rho} \leq \frac{23}{27}, \quad r=1, \ldots, M .
\end{aligned}
$$

The Courant-Fisher min-max theorem [11, Thm. 4.2.6] and Weyl's perturbation theorem [11, Thm. 4.3.1] finally yield:

$$
\operatorname{cond}(A) \leq \operatorname{cond}(Q) \cdot \max _{j} \operatorname{cond}\left(A_{j}\right) \leq \frac{5}{\tau} .
$$

Altogether, the improved variant of this technique can be used for nearly colliding pairs, but leads to a stronger assumption on $\rho$ for all moderate uniformity constants $c$.

\section{Numerical examples}

All computations were carried out using MATLAB R2019b. As a test for the bounds in the case of one pair of nearly colliding nodes, we use the following configuration. Let the number of nodes $M=20$ and $M=200$ be fixed, respectively. Moreover, we choose $N=1+12(M-1)$ which ensures that all nodes fit on the unit interval. We choose $\tau \in\left[10^{-11}, 1\right]$ logarithmically uniformly at random and $\rho_{3}, \ldots, \rho_{M} \in$ $[6,12]$ uniformly at random. Then, we set the nodes $t_{1}<\cdots<t_{M} \in[0,1)$ such that $t_{1}=0, t_{2}=\tau / N$ and for $j=3, \ldots, M,\left|t_{j}-t_{j-1}\right|=\rho_{j} / N$. Afterwards, the condition number of the corresponding Vandermonde matrix is computed. This procedure is repeated 100 times and the results are presented in Fig. 6 (left).

For pairs of nearly colliding nodes, we use the following configuration. Let the number of nodes $M=20$ and $M=200$ be fixed, respectively. Moreover, we choose the parameter $c=2$ and $\tau_{\max }$ and $\rho_{\min }$ as in Theorem 4.7. To ensure that all nodes fit on the unit interval, we choose $N$ as the smallest odd integer bigger than $\left(c \tau_{\max }+2 \rho_{\min }\right) M / 2$. Then, we choose $\tau \in\left[10^{-11}, 1\right]$ logarithmically uniformly at 

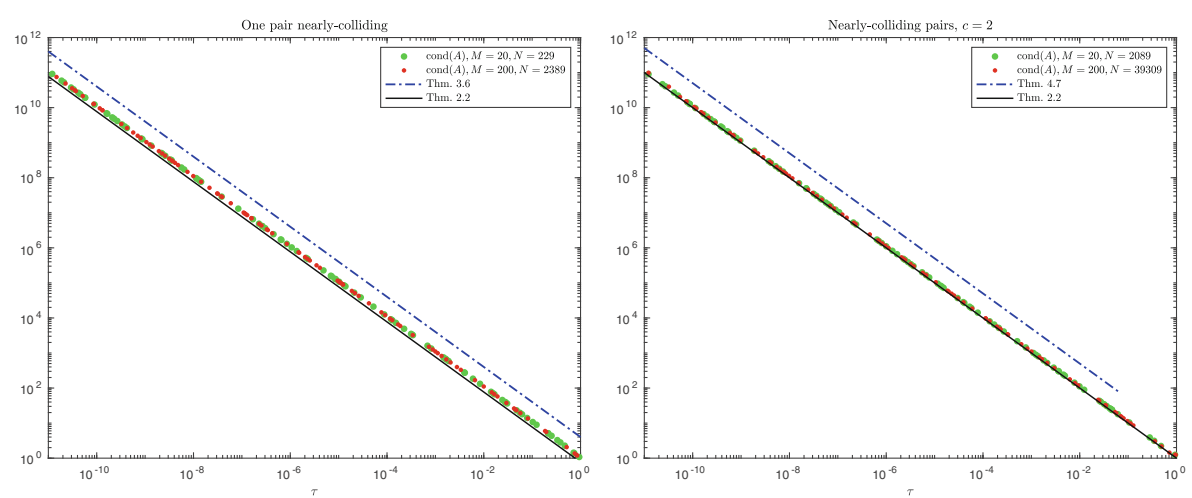

Fig. 6 Numerical experiments for bounds on the condition number, lower bounds from Theorem 2.2; left, one nearly colliding pair, upper bound from Theorem 3.6; right, pairs of nearly colliding nodes, upper bound from Theorem 4.7

random and set the nodes $t_{1}<\cdots<t_{M} \in[0,1)$ such that $t_{1}=0, t_{2}=\tau / N$ and for $j=3, \ldots, M,\left|t_{j}-t_{j-1}\right|=\rho_{j} / N$ if $j$ is odd or $\left|t_{j}-t_{j-1}\right|=\tau_{j} / N$ if $j$ is even, where $\tau_{j} \in[\tau, c \tau]$ and $\rho_{j} \in\left[\rho_{\min }, 2 \rho_{\min }\right]$ are picked uniformly at random, respectively. Afterwards, the condition number of the corresponding Vandermonde matrix is computed. This procedure is repeated 100 times and the results are presented in Fig. 6 (right). Note that Theorem 4.7 makes the restriction $\tau \leq \tau_{\max }=\frac{1}{4}$, which seems to be an artifact of our proof technique.

In order to compare Theorem 4.7 with the results from [4, Cor. 3.6], we need to satisfy the assumptions of both results. We take $M=3$ nodes with two nodes nearly colliding, i.e., $t_{1}=0, t_{1}=\tau / N$ and $t_{2}=t_{1}+\rho / N$. The assumptions in [4, Cor. 3.6] make it necessary that the nodes lie on an interval of length $\frac{1}{2 M^{2}}=\frac{1}{18}$. We choose the parameter $c=1, \rho_{\min }=12$, and $N=1001$. Then, we pick $\tau \in$ $\left[10^{-11}, 1\right] \operatorname{logarithmically}$ uniformly at random and $\rho \in\left[\rho_{\min }, \frac{N}{2 M^{2}}-\tau\right]$ uniformly at random. Afterwards, the inverse of the smallest singular value (norm of MoorePenrose pseudo inverse) of the corresponding Vandermonde matrix is computed. This procedure is repeated 100 times and the results normalized by $\tau \sqrt{N}$ are presented in Fig. 7 (left). From [4, Cor. 3.6], we get:

$$
\left\|A^{\dagger}\right\| \leq \frac{2(2 \pi)^{M-1} M^{2 M-1}}{\pi} \cdot \frac{N \sqrt{N}}{(N-1) \sqrt{N-1}} \cdot \frac{1}{\tau \sqrt{N}} \approx 6116 \cdot \frac{1}{\tau \sqrt{N}}
$$

for $\tau \leq 1$, whereas Theorem 4.7 provides $\left\|A^{\dagger}\right\| \leq \sqrt{11.3} \cdot \frac{1}{\tau \sqrt{N}} \approx 3.4 \cdot \frac{1}{\tau \sqrt{N}}$ for $\tau \leq \frac{1}{4}$.

In order to compare our results with the ones from the second version of [16, Thm. 1, Thm. 2], we set the parameter $N=2^{15}+1, c=1$ and $M=4$ and $M=20$, respectively. All pairs of nodes are placed uniformly, such that $t_{j}=\frac{2 j-2}{M}$ and $t_{j+M / 2}=t_{j}+\frac{\tau}{N}$ for $j=1, \ldots, \frac{M}{2}$, where $\tau$ is picked logarithmically uniformly at random from $\left[10^{-11}, 1\right]$. Afterwards, the inverse of the smallest singular value (norm of Moore-Penrose pseudo inverse) of the corresponding Vandermonde matrix 

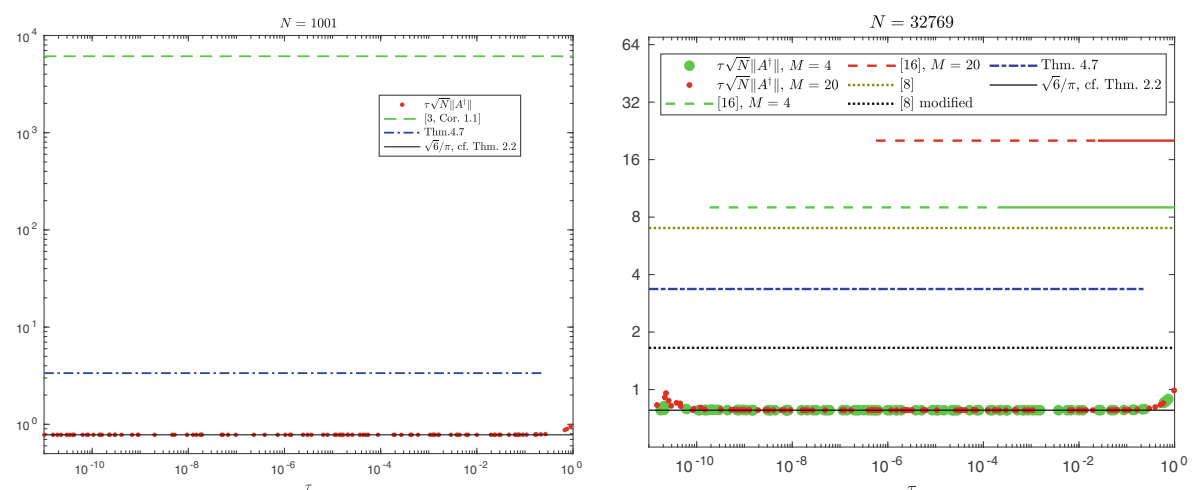

Fig. 7 Upper bounds for $\left\|A^{\dagger}\right\|$. Left, Comparison of Thm. 4.7 with [4, Cor. 3.6]; right, comparison of Thm. 4.7 with [16, Thm. 2], [8, Cor. 4.2] and its modification for our setting as mentioned in Remark 4.13

is computed. This procedure is repeated 100 times and the results normalized by $\tau \sqrt{N}$ are presented in Fig. 7 (right). Note that [16, Thm. 1, ineq. (2.2), Thm. 2, ineq. (2.5)] restricts:

$$
\tau \geq \frac{20^{2} M 2^{5} N^{3}}{\rho^{2}(N-1)^{3}} \approx\left\{\begin{array}{l}
1.9 \cdot 10^{-4}, \\
2.4 \cdot 10^{-2},
\end{array} \quad \tau \geq \frac{10^{4} 2^{10} M N^{5}}{\rho^{4} \pi(N-1)^{5}} \approx\left\{\begin{array}{c}
1.8 \cdot 10^{-10}, \quad M=4, \\
5.6 \cdot 10^{-7}, \quad M=20,
\end{array}\right.\right.
$$

respectively, where we used the uniform bound $\rho<\frac{2 N}{M}$. The results are shown in Fig. 7 (right) by proper lines [16, Thm. 2, ineq. (2.5)] and by broken lines [16, Thm. 1, ineq. (2.2)]. In both cases and with minor corrections, the resulting estimate is:

$$
\begin{aligned}
\left\|A^{\dagger}\right\| & \leq \frac{20 \sqrt{2}}{19}\left(1-\frac{\pi^{2}}{12}\right)^{-1 / 2} \frac{N-1}{2}\left\lfloor\frac{N-1}{2}\right\rfloor^{-1} \frac{4}{\pi} \sqrt{M} \frac{\sqrt{N}}{\sqrt{N-1}} \cdot \frac{1}{\tau \sqrt{N}} \\
& \approx \begin{cases}9 \cdot \frac{1}{\tau \sqrt{N}}, & M=4, \\
20.1 \cdot \frac{1}{\tau \sqrt{N}}, & M=20,\end{cases}
\end{aligned}
$$

whereas Theorem 4.7 provides again $\left\|A^{\dagger}\right\| \leq 3.4 \cdot \frac{1}{\tau \sqrt{N}}$ for $\tau \leq \frac{1}{4}$. We note that our bound remains valid for $c>1$ but the restriction on $\tau$ becomes more severe.

\section{Summary}

We proved upper and lower bounds for the spectral condition number of rectangular Vandermonde matrices with nodes on the complex unit circle. If pairs of nodes nearly collide, the studied condition number grows linearly with the inverse separation distance. In contrast to the more general results $[4,16]$, we provide reasonable sharp and absolute constants but have to admit that our technique most likely will not generalize to more than two nodes nearly colliding. Note that our easy to achieve lower bound seems to capture the situation more accurately than the upper bound. We posed mild technical conditions in our proofs, which cannot be confirmed to be necessary numerically. While [4] provided the right growth order for the first 
time, some of the imposed conditions are very restrictive and the involved constants are quite pessimistic. The second version of [16] provided a quite general framework and presented decent results with only a mild artificial growth of the condition number with respect to the number of nodes. Moreover, a technical condition there prevents the separation distance from going to zero for a fixed number of nodes and a fixed bandwidth. We believe that both problems can be fixed at least partially and thus [16] seems to be a good framework for understanding node configurations with nearly colliding nodes. Recently, the manuscript [8] came to our attention-it considers pairs of nearly colliding nodes and weakens the assumptions considerably and gives, after modifications, stronger bounds on the smallest singular value. The taken approach differs completely from ours and the ones in $[4,16]$, but rather generalizes the construction of [18] to pairs of nearly colliding nodes.

Acknowledgments Open Access funding provided by Projekt DEAL. The authors thank both referees for their valuable suggestions.

Funding The authors received support from the projects DFG-GK1916 and DFG-SFB944.

Open Access This article is licensed under a Creative Commons Attribution 4.0 International License, which permits use, sharing, adaptation, distribution and reproduction in any medium or format, as long as you give appropriate credit to the original author(s) and the source, provide a link to the Creative Commons licence, and indicate if changes were made. The images or other third party material in this article are included in the article's Creative Commons licence, unless indicated otherwise in a credit line to the material. If material is not included in the article's Creative Commons licence and your intended use is not permitted by statutory regulation or exceeds the permitted use, you will need to obtain permission directly from the copyright holder. To view a copy of this licence, visit http://creativecommonshorg/licenses/by/4. $0 /$.

\section{Appendix A}

The following technical results are used within the proofs of our main results.

Lemma A.1 Let $n \in \mathbb{N}, N=2 n+1$, then the Dirichlet kernel (2.2) is bounded by

$$
N-\frac{\pi^{2}}{6} N^{3} t^{2} \leq D_{n}(t) \leq N-N^{3} t^{2}, \quad 0 \leq|t| \leq \frac{1}{N} .
$$

Furthermore, the Dirichlet kernel and its first two derivatives are bounded by

$$
\begin{aligned}
\left|D_{n}(t)\right| & \leq \frac{1}{2|t|} \\
\left|D_{n}^{\prime}(t)\right| & \leq N^{2}\left(\frac{\pi}{2 N|t|}+\frac{1}{2 N^{2}|t|^{2}}\right) \\
\left|D_{n}^{\prime \prime}(t)\right| & \leq N^{3}\left(\frac{\pi^{2}}{2 N|t|}+\frac{\pi}{N^{2}|t|^{2}}+\frac{1}{N^{3}|t|^{3}}\right)
\end{aligned}
$$

for $0<|t| \leq 1 / 2$. 
Proof Due to symmetry, it suffices to prove all bounds for $t>0$ and we use the explicit expression of the Dirichlet kernel in (2.2). The lower bound on $t$ can be derived from the inequalities $x-x^{3} / 6 \leq \sin (x) \leq x$, that hold for all $x \in[0, \pi]$. The left inequality with $x=N \pi t$ and the right inequality with $x=\pi t$ lead to:

$$
\sin (N \pi t) \geq\left(N-\frac{\pi^{2}}{6} N^{3} t^{2}\right) \pi t \geq\left(N-\frac{\pi^{2}}{6} N^{3} t^{2}\right) \sin (\pi t) .
$$

The upper bound on $D_{n}(t)$ can be derived from the inequality $\cos (\alpha x) \leq \cos (x)$ that holds for all $x \in[0, \pi / 2]$ and $\alpha>1$ such that $\alpha x \in[0, \pi / 2]$. Integrating this inequality, choosing $\alpha=N / 2$ and $x=\pi t$, and applying the double angle formula yields:

$$
\frac{\sin (N \pi t)}{2 \cos \left(\frac{N}{2} \pi t\right)}=\sin \left(\frac{N}{2} \pi t\right) \leq \frac{N}{2} \sin (\pi t) .
$$

Reordering the inequality and applying that $\cos (x) \leq 1-4 x^{2} / \pi^{2}$ for all $x \in[0, \pi / 2]$ yields:

$$
\frac{\sin (N \pi t)}{\sin (\pi t)} \leq N \cos \left(\frac{N}{2} \pi t\right) \leq N\left(1-N^{2} t^{2}\right) .
$$

Finally, the remaining bounds on the absolute values can be proven by calculating the first and second derivatives and using $\sin (x) \geq 2 x / \pi$ and $\cot (x) \leq 1 / x$ that hold for all $x \in(0, \pi / 2]$.

Lemma A.2 Let $M, \tilde{M} \in \mathbb{C}^{m \times n}$ with $\left|M_{k \ell}\right| \leq \tilde{M}_{k \ell}$ for all $k=1, \ldots, m$, $\ell=$ $1, \ldots, n$, then:

$$
\|M\| \leq\|\widetilde{M}\|
$$

Proof We directly show the result by:

$$
\begin{aligned}
\|M\|^{2} & =\max _{\|x\|=1}\|M x\|^{2}=\max _{\|x\|=1} \sum_{k=1}^{m}\left|\sum_{\ell=1}^{n} M_{k \ell} x_{\ell}\right|^{2} \leq \max _{\|x\|=1} \sum_{k=1}^{m}\left(\sum_{\ell=1}^{n}\left|M_{k \ell}\right|\left|x_{\ell}\right|\right)^{2} \\
& \leq \max _{\|x\|=1} \sum_{k=1}^{m}\left(\sum_{\ell=1}^{n} \tilde{M}_{k \ell}\left|x_{\ell}\right|\right)^{2}=\max _{\|x\|=1} \sum_{k=1}^{m}\left(\sum_{\ell=1}^{n} \tilde{M}_{k \ell} x_{\ell}\right)^{2}=\|\tilde{M}\|^{2} .
\end{aligned}
$$

Note that similar estimates can be found for the Frobenius norm in [11, p. 520].

Lemma A.3 (Norm of matrix inverse) Let $M \in \mathbb{C}^{n \times n}$ Hermitian and positive definite and $I \in \mathbb{C}^{n \times n}$ the identity matrix. Let $\eta \in \mathbb{R}$ be a parameter satisfying $\eta>\|M\|$, then:

$$
\left\|M^{-1}\right\|=\frac{1}{\eta-\|\eta I-M\|}
$$

Proof Since $M$ is positive definite, let its real, positive eigenvalues be given by $\lambda_{1}(M) \geq \cdots \geq \lambda_{n}(M)>0$. By assumption $\eta>\|M\|=\lambda_{\max }(M)$ and 
therefore, $\eta I-M$ is positive definite as well with largest eigenvalue $\lambda_{\max }(\eta I-M)=$ $\eta-\lambda_{\min }(M)$. This finally leads to:

$$
\left\|M^{-1}\right\|=\frac{1}{\lambda_{\min }(M)}=\frac{1}{\eta-\left(\eta-\lambda_{\min }(M)\right)}=\frac{1}{\eta-\lambda_{\max }(\eta I-M)}=\frac{1}{\eta-\|\eta I-M\|} .
$$

Lemma A.4 (Schur complement, cf. [11, eq. (0.8.5.3)]) Let $n_{1}, n_{2} \in \mathbb{N}$ and the matrix $M \in \mathbb{C}^{\left(n_{1}+n_{2}\right) \times\left(n_{1}+n_{2}\right)}$ be a $2 \times 2$ block matrix of the form:

$$
M=\left(\begin{array}{ll}
M_{1} & M_{2} \\
M_{3} & M_{4}
\end{array}\right), M_{1} \in \mathbb{C}^{n_{1} \times n_{1}}, M_{4} \in \mathbb{C}^{n_{2} \times n_{2}},
$$

with $M_{1}$ being invertible. Then, the Schur complement decomposition is given by:

$$
M=\left(\begin{array}{cc}
I_{n_{1}} & 0 \\
-M_{3} M_{1}^{-1} & I_{n_{2}}
\end{array}\right)^{-1}\left(\begin{array}{cc}
M_{1} & 0 \\
0 & M_{4}-M_{3} M_{1}^{-1} M_{2}
\end{array}\right)\left(\begin{array}{cc}
I_{n_{1}} & -M_{1}^{-1} M_{2} \\
0 & I_{n_{2}}
\end{array}\right)^{-1} .
$$

The block $\left[M / M_{1}\right]:=M_{4}-M_{3} M_{1}^{-1} M_{2}$ is called Schur complement of $M_{1}$ in $M$.

Lemma A.5 (Cauchy interlacing theorem for eigenvalues, cf. [11, Thm. (4.3.28)]) Let $M \in \mathbb{C}^{n \times n}$ be a Hermitian complex matrix, such that:

$$
M=\left(\begin{array}{ll}
M_{1} & M_{2} \\
M_{2}^{*} & M_{3}
\end{array}\right), \quad M_{1} \in \mathbb{C}^{m \times m}, M_{2} \in \mathbb{C}^{m \times(n-m)}, M_{3} \in \mathbb{C}^{(n-m) \times(n-m)} .
$$

Let the eigenvalues of $M$ and $M_{1}$ be ordered in non-decreasing order, then:

$$
\lambda_{i}(M) \leq \lambda_{i}\left(M_{1}\right) \leq \lambda_{i+n-m}(M), \quad i=1, \ldots, m .
$$

Lemma A.6 (Block Gerschgorin theorem, cf. [11, 6.1.P17] or [10, Thm. 5]) Let $M \in \mathbb{C}^{n m \times n m}$ be an $m \times m$ block matrix with blocks $M_{i k} \in \mathbb{C}^{n \times n}$. Let the diagonal blocks $M_{i i}$ be normal and denote $\lambda_{1}^{(i)}, \ldots, \lambda_{n}^{(i)}$ their eigenvalues, respectively. Then, the eigenvalues of $M$ are included in the set:

$$
\bigcup_{i=1}^{n} \bigcup_{j=1}^{m}\left\{z \in \mathbb{C}:\left|z-\lambda_{j}^{(i)}\right| \leq \sum_{k \neq i}\left\|M_{i k}\right\|\right\} .
$$

In particular, we have for $M \in \mathbb{C}^{m \times n}$ the inequalities:

$$
\left\|\left(\begin{array}{cc}
0 & M^{*} \\
M & 0
\end{array}\right)\right\| \leq\|M\| \text { and } \quad\left\|\left(\begin{array}{cc}
I & 0 \\
M & I
\end{array}\right)\right\|^{2} \leq 1+\|M\|+\|M\|^{2} .
$$

\section{References}

1. Akinshin, A., Goldman, G., Yomdin, Y.: Geometry of error amplification in solving Prony system with near-colliding nodes. arXiv:1701.04058 (2017)

2. Aubel, C., Bölcskei, H.: Vandermonde matrices with nodes in the unit disk and the large sieve. Appl. Comput. Harmon. Anal. 47(1), 53-86 (2019) 
3. Batenkov, D., Diederichs, B., Goldman, G., Yomdin, Y.: The spectral properties of Vandermonde matrices with clustered nodes (2019)

4. Batenkov, D., Demanet, L., Goldman, G., Yomdin, Y.: Conditioning of partial nonuniform Fourier matrices with clustered nodes. SIAM J. Matrix Anal. Appl. 41(1), 199-220 (2020). arXiv:1909.01927

5. Bazán, F.S.V.: Conditioning of rectangular Vandermonde matrices with nodes in the unit disk. SIAM J. Matrix Anal. Appl. 21(2), 679-693 (1999)

6. de Prony, B.GR.: Essai éxperimental et analytique: sur les lois de la dilatabilité de fluides élastique et sur celles de la force expansive de la vapeur de l'alkool, a différentes températures. J. l'École Polytech. 1(22), 24-76 (1795)

7. Demanet, L., Nguyen, N.: The recoverability limit for superresolution via sparsity ArXiv e-prints. arXiv:1502.01385 (2015)

8. Diederichs, B.: Well-posedness of sparse frequency estimation. arXiv:1905.08005 (2019)

9. Donoho, D.L.: Superresolution via sparsity constraints. SIAM J. Math. Anal. 23(5), 1309-1331 (1992)

10. Feingold, D.G., Varga, R.S.: Block diagonally dominant matrices and generalizations of the gerschgorin circle theorem. Pac. J. Math. 12(4), 1241-1250 (1962)

11. Horn, R.A., Johnson, C.R.: Matrix Analysis, 2nd edn. Cambridge University Press, New York (2013)

12. Hua, Y., Sarkar, T.K.: Matrix pencil method for estimating parameters of exponentially damped/undamped sinusoids in noise. IEEE Trans. Acoust. Speech Signal Process. 38(5), 814-824 (1990)

13. Kunis, S., Nagel, D.: On the smallest singular value of multivariate vandermonde matrices with clustered nodes. Linear Algebra and its Applications 604, 1-20 (2020). ArXiv e-prints

14. Kunis, S., Potts, D.: Stability results for scattered data interpolation by trigonometric polynomials. SIAM J. Sci. Comput. 29, 1403-1419 (2007)

15. Kunis, S., Möller, H.M., Peter, T., von der Ohe, U.: Prony's method under an almost sharp multivariate Ingham inequality. J. Fourier Anal. Appl. 24(5), 1306-1318 (2018)

16. Li, W., Liao, W.: Stable super-resolution limit and smallest singular value of restricted Fourier matrices. arXiv:1709.03146 (2017)

17. Liao, W., Fannjiang, A.: MUSIC for single-snapshot spectral estimation: stability and superresolution. Appl. Comput. Harmon. Anal. 40(1), 33-67 (2016)

18. Moitra, A.: Super-resolution, extremal functions and the condition number of Vandermonde matrices. In: STOC'15-Proceedings of the 2015 ACM Symposium on Theory of Computing, pp. 821-830. ACM, New York (2015)

19. Morgenshtern, V.I., Candès, E.J.: Super-resolution of positive sources: the discrete setup. SIAM J. Imaging Sci. 9(1), 412-444 (2016)

20. Potts, D., Tasche, M.: Error estimates for the ESPRIT algorithm. In: Large Truncated Toeplitz Matrices, Toeplitz Operators, and Related Topics, volume 259 of Oper. Theory Adv. Appl., p. Cham. Birkhäuser/Springer (2017)

21. Roy, R., Kailath, T.: ESPRIT-estimation of signal parameters via rotational invariance techniques. IEEE Trans. Acoust. Speech Signal Process. 37(7), 984-995 (1989)

22. Schmidt, R.O.: Multiple emitter location and signal parameter estimation. IEEE Trans. Antennas Propag. 34(3), 276-280 (1986)

Publisher's note Springer Nature remains neutral with regard to jurisdictional claims in published maps and institutional affiliations. 